\title{
A SUBGROUP THEOREM FOR FREE NILPOTENT GROUPS
}

\author{
BY \\ S. MORAN
}

In our study of countable subgroups of the unrestricted $n$th nilpotent product of infinite cycles $[10 ; 11]\left({ }^{1}\right)$, it became necessary to have some information concerning the structure of subgroups of free nilpotent groups. This is available for free second nilpotent groups in Golovin and Goldina [2]. Karl Gruenberg drew our attention to the paper of Goldina [1], where a subgroup theorem for free nilpotent groups is stated, but no proofs are given. Below we give a corrected version of this paper with proofs $\left({ }^{2}\right)$. Every subgroup $B$ of a free $n$th nilpotent group can be generated by a set of $n$ subgroups

$$
B_{1}, B_{2}, \cdots, B_{l}, \cdots, B_{n}
$$

which are free nilpotent groups of $\operatorname{class}\left({ }^{8}\right)$

$$
n,\left[\frac{n}{2}\right], \cdots,\left[\frac{n}{l}\right], \cdots, 1
$$

respectively. This fact, in conjunction with certain other conditions which state how the subgroups $B_{1}, B_{2}, \cdots, B_{n}$ are related among themselves $\left({ }^{4}\right)$, characterize the subgroups of free $n$th nilpotent groups. As an example of this characterization we have that if $B$ is a group satisfying these conditions and

$$
B_{1}, B_{2}, \cdots, B_{n-1}
$$

have finite ranks, then $B$ can be mapped isomorphically into a free $n$th nilpotent group. In proving these results, we found it necessary to employ techniques due to $\mathrm{Mal}^{\prime} \mathrm{cev}$ which are contained in a well-known paper of Lazard [7], where a process is given for embedding a torsion-free nilpotent group in a nilpotent Lie algebra over the rationals. An important role is played by a fundamental theorem of Siršov [13] on subalgebras of free Lie algebras over a field. Also we generalize the concept of a basic commutator by distinguishing between original and nonoriginal commutators.

We adopt the following notation. Let $G$ be an arbitrary group and ${ }^{n} G$ the $n$th member of the lower central series of $G$ associated with the commutator $\left[\left[\cdots\left[x_{1}, x_{2}\right], \cdots\right], x_{n}\right]$. If ${ }^{n+1} G=1$, while ${ }^{n} G \neq 1$, then $G$ is said to be nil-

Received by the editors June 19, 1961.

(1) Cf. note at end of [10]. In the forthcoming paper [11], we will also consider the unrestricted third Burnside product both of infinite cycles and of cycles of order 3.

(2) The main subgroup theorems of Goldina [1], Theorems 4 and 5, are incorrect.

( ${ }^{3}$ If $r$ is a positive real number, then $[r]$ denotes the integral part of $r$.

(4) Cf. Theorem 3.4 and Theorem 3.7. 
potent of $\left(^{(5)}\right.$ class $n$. If $\mathcal{F}$ denotes a free group, then $\mathscr{F} /{ }^{n+1} \mathcal{F}$ is known as a free $n$th nilpotent group. A set of free generators of $\mathcal{F}$ gives rise, in a natural way, to a set of relatively free generators of $\mathcal{F} /{ }^{n+1} \mathcal{F}$, which we shall also call a set of free generators of $\mathscr{F} /{ }^{n+1} \mathcal{F}$. Finally we recall the definition of basic commutators, which give a unique representation for the elements of $\mathscr{F} /{ }^{n+1} \mathfrak{F}$. Let $a_{1}, a_{2}, \ldots$ be an ordered collection of elements of an arbitrary group. They are said to be basic commutators of weight 1 . Suppose that the basic commutators of weight less than $l$ on $a_{1}, a_{2}, \cdots$ have already been defined and ordered in such a way that

"basic commutator of weight $s$ " < "basic commutator of weight $t$,"

if $s<t$. Now $b$ is said to be a basic commutator of weight $l$ if $b=\left[b^{\prime}, b^{\prime \prime}\right]$, where

(i) $b^{\prime}$ and $b^{\prime \prime}$ are basic commutators of weight $s$ and $t$ respectively;

(ii) $s+t=l$ and $b^{\prime}>b^{\prime \prime}$;

(iii) if $b^{\prime}=\left[b_{1}^{\prime}, b_{2}^{\prime}\right]$, where $b_{1}^{\prime}$ and $b_{2}^{\prime}$ are basic commutators, then $b^{\prime \prime} \geqq b_{2}^{\prime}$. The fundamental basis theorem of M. Hall [3] and P. Hall [5] states that every element of $\mathfrak{F} /{ }^{n+1} \mathcal{F}$ can be represented uniquely in the form

$$
b_{1}^{m_{1}} b_{2}^{m_{2}} \cdots b_{N}^{m_{N}}
$$

where the $b_{i}$ are basic commutators on a set of free generators of $\mathcal{F} /{ }^{n+1} \mathfrak{F}$ of weight less than $n+1$ and where $b_{i}<b_{j}$ if $i<j$. Further it is of value to note that the basic commutators of weight $n$ form a basis for the centre, ${ }^{n \mathcal{F}} /{ }^{n+1} \mathcal{F}$, of $\mathcal{F} /{ }^{n+1} \mathcal{F}$.

1. Concerning subgroups of free nilpotent groups. The statements of Lemma 1.1 and Theorems 1.3 and 1.6 given below are contained in the paper of Goldina [1].

LEMMA 1.1. In a free nth nilpotent group $G$ the basic commutators of weight $l$ freely generate a free nilpotent group ${ }^{(8)}$ of class $[n / l]$.

Proof. Obviously $G_{l}$ is a subgroup of ${ }^{l} G$, which is nilpotent of class less than or equal to $k$, where $k$ is given by

$$
\frac{n-l+1}{l} \leqq k<\frac{n+1}{l} \text {. }
$$

It is easy to see that $k=[n / l]$. The required result follows from the fact that the basic commutators formed from "the basic commutators of weight $l$ " are basic commutators in $G$.

It is now possible to give a generalization of a theorem of Mal'cev [8]. This states $\left({ }^{7}\right)$ that a set of elements of $G$ which is linearly independent

(5) Goldina [1] seems to call such a group nilpotent of class $n+1$ and $\mathcal{F} /{ }^{n+1} \mathfrak{F}$ is accordingly a free $(n+1)$ th nilpotent group.

( $)$ If $G$ is of rank two, then $G$ has only one basic commutator of weight 1 . Hence, in this particular case, the basic commutators of weight 1 generate an infinite cyclic subgroup.

( 7 Cf. Mostowski [12] for a group theoretical proof of this result. 
modulo ${ }^{2} G$ freely generates a free $n$th nilpotent subgroup of $G$. However, we first introduce a simple consequence of Hall's commutator collecting process [4].

Lemma 1.2. Let $b_{\alpha}, \alpha \in M$, be a set of elements of the lth member of the lower central series of an arbitrary group $G$ and

$$
b_{\alpha}=c_{\alpha} \cdot d_{\alpha},
$$

where $c_{\alpha} \in \in^{l} G, c_{\alpha} \bigoplus^{l+1} G$ and $d_{\alpha} \in^{l+1} G, d_{\alpha} \notin^{l} G$ for all $\alpha$ of $M$. Then for any complex $\left(^{8}\right)$ commutator $f\left(b_{\alpha_{1}}, b_{\alpha_{2}}, \cdots\right)$ of the elements $b_{\alpha}, \alpha \in M$, we have that

$$
f\left(b_{\alpha_{1}}, b_{\alpha_{2}}, \cdots\right)=f\left(c_{\alpha_{1}}, c_{\alpha_{2}}, \cdots\right) \bmod ^{l \omega+1} G,
$$

where $\omega$ is the weight of the complex commutator $f$.

TheOREM 1.3. A set of elements of a free nth nilpotent group $G$, which is contained in ${ }^{\prime} G$ and is linearly independent modulo ${ }^{l+1} G$, freely generate a free nilpotent subgroup of class $[n / l]$.

Proof. If $b_{\alpha}, \alpha \in M$, is a set of elements of ${ }^{l} G$ which is linearly independent modulo ${ }^{l+1} G$, then

$$
b_{\alpha}=c_{\alpha} \cdot d_{\alpha},
$$

where $c_{\alpha} \in G_{1}$ and $c_{\alpha} \bigoplus^{l+1} G$ and $d_{\alpha} \in^{l+1} G$ for all $\alpha$ of $M$. Form the basic commutators of weight $k$ on the elements $b_{\alpha}, \alpha \in M$, then any relation among them modulo ${ }^{l k+1} G$ gives rise, because of Lemma 1.2 , to a similar relation between the basic commutators of weight $k$ on the elements $c_{\alpha}, \alpha \in M$, Hence it is sufficient to show that the elements $c_{\alpha}, \alpha \in M$, freely generate a free nilpotent subgroup of class $[n / l]$. Now the elements $c_{\alpha}, \alpha \in M$, generate a subgroup of $G_{e}$, are linearly independent modulo ${ }^{l+1} G$ and hence also modulo $\left[G_{l}, G_{l}\right]$. Hence the required result follows from Lemma 1.1 and the above mentioned theorem of $\mathrm{Mal}^{\prime} \mathrm{cev}$. The converse to the above theorem is false.

EXAMPLE 1.4. Let $G$ be a free nilpotent group of class 10 and having the following set of free generators

$$
x_{1}, x_{2}, x_{3}, x_{4}, y_{1}, y_{2}, y_{3}, y_{4} \text {. }
$$

It is easy to see that the four commutators

$$
\left[\left[\left[x_{1}, y_{1}\right], y_{1}\right], y_{1}\right], \quad\left[\left[\left[x_{2}, y_{2}\right], y_{2}\right], y_{2}\right], \quad\left[\left[\left[\left[x_{3}, y_{3}\right], y_{3}\right], y_{3}\right],\right] y_{3}
$$

and

$$
\left[\left[\left[\left[x_{4}, y_{4}\right], y_{4}\right], y_{4}\right], y_{4}\right]
$$

freely generate a free second nilpotent subgroup in $G$.

Information about abelian subgroups of a free nilpotent group is given below.

(8) Cf. P. Hall [4]. 
THEOREM 1.5. Every abelian subgroup of a free nilpotent group is free abelian.

Proof. We proceed by induction on the class of nilpotency of the free nilpotent group. The result is well known to be true for a free abelian group $\left({ }^{9}\right)$. Suppose that every abelian subgroup of a free $m$ th nilpotent group, where $m<n$, is free abelian. If $G$ is a free $n$th nilpotent group and $Z(G)$ is the centre of $G$, then by a result of Witt [14], $G / \mathcal{Z}(G)$ is a free $(n-1)$ th nilpotent group. Let $B$ be an abelian subgroup of $G$. Obviously $B \cdot \mathcal{Z}(G)$ is an abelian subgroup of $G, \mathrm{Z}(G)$ is free abelian $\left({ }^{10}\right)$ and, by the induction hypothesis, $B \cdot \mathrm{Z}(G) / \mathrm{Z}(G)$ is also free abelian. However, by a well-known result $\left({ }^{9}\right)$, an abelian group that is the extension of a free abelian group by a free abelian group is itself a free abelian group. Hence $B$, as a subgroup of a free abelian group, is free abelian.

THEOREM 1.6. If $G$ is a free nth nilpotent group, then the lth member of the lower central series of $G$ is abelian if and only $\left({ }^{11}\right)$ if

$$
l>n / 2 \text {. }
$$

If this inequality holds, then ${ }^{l} G$ is a free abelian group of rank equal to the sum of the ranks of the free abelian groups

$$
{ }^{l} G /{ }^{l+1} G, \quad{ }^{l+1} G /{ }^{l+2} G, \cdots,{ }^{n} G .
$$

In fact, we have that ' $G$ is freely generated by the basic commutators of weight greater than or equal to $l$.

Proof. If ${ }^{l} G$ is abelian, then $G_{l}$ is abelian and it follows from Lemma 1.1 that $n / l<2$, that is, $l>n / 2$. The only exception to this is given in the footnote $\left.{ }^{11}\right)$. Conversely, if $l>n / 2$, then ${ }^{l} G$ is nilpotent of class less than or equal to $n / l<2$, that is, ${ }^{l} G$ is abelian and hence free abelian by Theorem 1.5. In fact, ${ }^{l} G$ has a basis consisting of all the basic commutators of weight greater than or equal to $l$.

Finally we notice that under the natural homomorphism of a free nilpotent group onto a free nilpotent group of the same rank but smaller class, certain free nilpotent subgroups are mapped onto free nilpotent subgroups. This is shown by the following theorem.

THEOREM 1.7. Let $B$ be a free kth nilpotent subgroup of a free nth nilpotent group $G$ which is contained in ' $G$ and freely generated by elements of $B$ that are linearly independent modulo ${ }^{l+1} G$. Further let $\phi$ be the natural homomorphism of $G$ onto a free mth $(m \leqq n)$ nilpotent group $G \phi$ which maps free generators onto

( ${ }^{9}$ Cf. for instance Kuroš [6].

(10) Witt [14].

(11) There is one exception to this result. If $G$ has rank two and $n=4$, then $[G, G]$ is free abelian (cf. footnote $\left.{ }^{(6)}\right)$. 
free generators. Then $B \phi$ is the trivial group or a nilpotent group $\left({ }^{12}\right)$ of class $[\mathrm{m} / \mathrm{l}]$ of $G \boldsymbol{\phi}$. Free generators of $B$ are mapped onto free generators of $B \boldsymbol{\phi}$.

Proof. If $B$ is contained in the kernel of $\phi$, then $B \phi$ is the trivial group. ${ }^{m+1} G$ is the kernel of $\phi$. It is easy to see that as the free generators of $B$ are linearly independent modulo ${ }^{l+1} G$, their images are linearly independent modulo ${ }^{l+1}\left(G /{ }^{m+1} G\right)$. Further ${ }^{l} G$ is mapped onto ${ }^{l}\left(G /{ }^{m+1} G\right)$. Hence the required result follows from Theorem 1.3.

The above result does not hold in general for any free nilpotent subgroup of $G$. This is shown by the free second nilpotent subgroup given in Example 1.4.

2. Subalgebras of a free nilpotent Lie algebra over a field. Let $\Omega$ be a commutative ring with a unit element. $L$ is said to be a Lie $\Omega$-algebra if it is an $\Omega$-module with respect to addition and has a Lie product operation $(x, y)$ defined on it for every pair of elements $x$ and $y$ of $L$. The formation of the Lie product is a bilinear operation and satisfies the identical relations

$$
(x, x)=0
$$

and

$$
((x, y), z)+((y, z), x)+((z, x), y)=0 .
$$

We can construct the lower central series for $L$ in the following manner. ${ }^{1} L=L$. Suppose that ${ }^{l} L$ has already been defined. Then ${ }^{l+1} L$ is the $\Omega$-subalgebra generated by all Lie products of the form $(x, y)$, where $x$ and $y$ belong to ${ }^{l} L$ and $L$ respectively. A Lie $\Omega$-algebra is said to be nilpotent of class $n$ if ${ }^{n+1} L=0$ while ${ }^{n} L \neq 0$.

Suppose that $L$ has a set of generators $a_{\alpha}, \alpha \in M$, and there are no relations between these generators except those that follow from the above laws for a Lie algebra. Then $L$ is said to be a free Lie $\Omega$-algebra. Such a free Lie $\Omega$-algebra can be constructed as a quotient $\Omega$-algebra of the free nonassociative $\Omega$-algebra on $a_{\alpha}, \alpha \in M$.

We can now define a free $n$th nilpotent Lie $\Omega$-algebra $\mathscr{L}$ to be $L /{ }^{n+1} L$, where $L$ is a free $\Omega$-algebra. For the rest of this paper our attention will be confined to the particular case when $\Omega$ is a field.

Lazard $[7, \S 4]$ studied the group $\left({ }^{13}\right)$ that can be obtained from $\mathscr{L}$ (over the rationals) if one defines multiplication by the Campbell-Hausdorff formula

$$
\Phi(x, y)=x+y+\frac{1}{2}(x, y)+\frac{1}{12}((x, y), y)+\frac{1}{12}((y, x), x)+\cdots .
$$

The group thus obtained is the $\mathrm{Mal}^{\prime}$ cev completion $M(G)$ of the free $n$th nilpotent group $G$ on the free generators $a_{\alpha}, \alpha \in M . M(G)$ is said to be the

(12) Infinite cyclic group if $B$ has rank 1.

(18) This group was first studied by Mal'cev (cf. bibliography in Lazard [7]). 
Mal'cev completion of a group $G$ if

(i) $G$ is a subgroup of $M(G)$;

(ii) $M(G)$ is a divisible torsion-free nilpotent group;

(iii) if $x$ is an arbitrary element of $M(G)$, then there exists a positive integer $n$ such that $x^{n}$ belongs to $G$.

We shall use certain properties of $\mathrm{Mal}^{\prime} \mathrm{cev}$ completions, an elegant account of which can be found in Lazard [7]. For instance, in $\mathcal{L}$ (over the rationals) which can also be considered to be $M(G)$, the concepts of subalgebra (ideal) and divisible subgroup (divisible normal subgroup) coincide. All homomorphisms of $\mathscr{L}$ are at the same time homomorphisms of $M(G)$.

It is possible to define the concept of a basic monomial in a Lie $\Omega$-algebra, using the Lie product $(x, y)$ instead of the commutator $[x, y]$. We can now state the famous

THEOREM 2.1. If $a_{\alpha}, \alpha \in M$, is a set of free generators of a free Lie $\Omega$-algebra $L$, then every element of $L$ can be expressed uniquely as a linear combination of basic monomials on $a_{\alpha}, \alpha \in M$.

Before stating the important Theorem of Siršov, we shall generalize the concept of a basic monomial by introducing original and nonoriginal monomials.

Definition 2.2. Let $L$ be a free Lie $\Omega$-algebra. A set of elements of $L$ which is linearly independent modulo ${ }^{2} L$ is both a set of original monomials of weight 1 and a set of nonoriginal monomials of weight 1 . We shall order this set arbitrarily. Suppose that all original and nonoriginal monomials of weight less than $l$ have already been constructed and ordered so that whenever $s<t$

$$
\text { "monomial of weight } s \text { " < "monomial of weight } t \text {." }
$$

$b$ is said to be a nonoriginal monomial of weight $l$ if it is a basic monomial on the original monomials of weight less than $l$ and $b$ belongs to ${ }^{\prime} L$ but $b$ does not belong to ${ }^{l+1} L$. Finally it remains to define original monomials of weight $l$. Let $L(l+1)$ denote the subalgebra $\left({ }^{14}\right)$ of $L$ generated by the above defined nonoriginal monomials of weight $l$ and ${ }^{l+1} L$. A set of elements of ${ }^{l} L$ which is linearly independent modulo $L(l+1)$ is called a set of original monomials of weight $l$.

This definition implies, in particular, that the original monomials of weight $l$ are linearly independent modulo ${ }^{l+1} L$. Of course, one can take the set of original monomials of some fixed weight to be empty. If we do this for all $l \geqq 2$, then we have the usual concept of a basic monomial.

THEOREM OF S̆IRŠOv 2.3. If $K$ is a subalgebra of a free Lie algebra $L$ over a field, then a set of original monomials of $L$ that generate $K$ is a set of free generators for $K$.

(14) It is easy to see that $L(l+1)$ is, in fact, an ideal. 
REMARK. The set of free generators for $K$ that Siršov [13] constructed differs from the one we have given above. This set of free generators depends on the terms of highest degree, while our set depends on the terms of lowest degree. Here we are referring to the unique representation given by the Theorem of Hall 2.1. However, the proof of the freeness of the above set of generators proceeds in exactly the same way as in Siršov [13], with the above change of emphasis from terms of highest degree to those of lowest degree.

CoRollary 2.3.1. A set of nonoriginal monomials of weight $l$ is linearly independent modulo ${ }^{l+1} L$.

We can now consider the above situation modulo ${ }^{n+1} L$. This gives rise to the following corresponding result for a free $n$th nilpotent Lie algebra $\mathscr{L}$ over a field.

COROLlary 2.3.2. A set of nonoriginal monomials of weight $l(\leqq n)$, of a free nth nilpotent Lie algebra $\mathfrak{L}$ over a field, is linearly independent modulo ${ }^{l+1} \mathfrak{L}$.

Notice that the original monomials are not uniquely determined by a subalgebra $K$ of $\mathscr{L}$ and that the same is, therefore, also true for the nonoriginal monomials of $K$. However, as is easily seen by the Theorem of Siršov, their number is uniquely determined by $K$.

Corollary 2.3.3. Let $K$ be a subalgebra of $\mathcal{L}$. The number $o_{i}$, in a maximal set of original monomials of weight $i(i=1,2, \cdots, n)$ in $K$, is uniquely determined by $K$. Further the number of nonoriginal monomials of weight l in $K$ is a uniquely determined function of $o_{1}, o_{2}, \cdots, o_{l-1}$ and $l$ only and is independent of the choice of the set of original monomials in $K$; it is a finite number if and only if all $o_{1}, o_{2}, \cdots, o_{l-1}$ are finite $(l=1,2, \cdots, n)$.

Using Theorem 3 of Witt [14], it is possible to give an explicit formula for the number of nonoriginal monomials of weight $l$, provided $o_{1}, o_{2}, \cdots, o_{l-1}$ are all finite $(l=1,2, \cdots, n)$.

3. Subgroup theorem for free nilpotent groups. By embedding a torsionfree nilpotent group in a nilpotent Lie algebra over the rationals, we shall now obtain a subgroup theorem. It will be necessary to use the apparatus developed in the previous paragraph.

THEOREM 3.1. Let $B$ be a subgroup of a free nth nilpotent group $G$. Then $B$ is generated by a set of $n$ subgroups

$$
B_{1}, B_{2}, \cdots, B_{n},
$$

where

(i) $B_{l}$ is a free nilpotent group $\left({ }^{15}\right)$ of class $[n / l](l=1,2, \cdots, n)$;

(15) We adopt the convention that a free $m$ th nilpotent group of rank 0 is the trivial group, while that of rank 1 is the infinite cyclic group. 
(ii) $\left[B_{i}, B_{j}\right] \leqq\left\{B_{i+j}, \cdots, B_{n}\right\}$, if $i+j \leqq n$, and

$$
\left[B_{i}, B_{j}\right]=1 \text { if } i+j>n \text {; }
$$

(iii) $\left\{B_{l}, B_{l+1}, \cdots, B_{n}\right\} /\left\{B_{l+1}, \cdots, B_{n}\right\}$ is a free abelian group freely generated by the images of the free generators of $B_{l}(l=1,2, \cdots, n-1)$.

Proof. To construct the subgroups $B_{l}$ we proceed as follows. Let $b_{\alpha(1)}$ denote a typical element of a set of elements of $B$ whose images form a basis for

$$
B \cdot{ }^{2} G /{ }^{2} G \text {. }
$$

Put $B_{1}$ equal to the subgroup of $G$ generated by the elements $b_{\alpha(1)}$; then

$$
B=B_{1} \cdot\left(B \cap{ }^{2} G\right) \text {. }
$$

Suppose that

$$
B_{1}, B_{2}, \cdots, B_{l-1}
$$

have already been constructed with

$$
B \cap{ }^{i} G=B_{i} \cdot\left(B \cap{ }^{i+1} G\right)
$$

for $i=1,2, \cdots, l-1$. Let $b_{\alpha(l)}$ denote a typical element of a set of elements of $B \cap^{\imath} G$ whose images form a basis for

$$
\left(B \cap{ }^{l} G\right) \cdot{ }^{l+1} G /{ }^{l+1} G .
$$

Put $B_{l}$ equal to the subgroup generated by the elements $b_{\alpha(l)}$; then

$$
B \cap{ }^{l} G=B_{l} \cdot\left(B \cap{ }^{l+1} G\right) .
$$

Hence we have that

$$
B=\left\{B_{1}, B_{2}, \cdots, B_{n}\right\} .
$$

By Theorem 1.3, $B_{l}$ is a free nilpotent group of class $[n / l]$, for $l=1,2, \cdots, n$. From the construction of the subgroups $B_{l}$, we have that

$$
\left[B_{i}, B_{j}\right] \leqq\left[{ }^{i} G,{ }^{i} G\right] \cap B \leqq{ }^{i+i} G \cap B .
$$

If $i+j>n$, then $\left[B_{i}, B_{j}\right]=1$, otherwise

Finally

$$
\left[B_{i}, B_{j}\right] \leqq\left\{B_{i+j}, \cdots, B_{n}\right\} .
$$

$$
\begin{aligned}
\left\{B_{l}, B_{l+1}, \cdots, B_{n}\right\} /\left\{B_{l+1}, \cdots, B_{n}\right\} & =\left(B \cap \cap^{l} G\right) /\left(B \cap{ }^{l+1} G\right) \\
& \cong\left(B \cap \cap^{l} G\right) \cdot{ }^{l+1} G /{ }^{l+1} G,
\end{aligned}
$$

by the isomorphism theorem, which gives the required result.

We now define, as in the previous paragraph, the concepts of original and nonoriginal commutators. We will do this in a group satisfying the above properties (i), (ii) and (iii) of Theorem 3.1. 
Definition 3.2. Let $B$ be a group generated by $n$ subgroups $B_{1}, B_{2}, \cdots$, $B_{n}$ that satisfy the above conditions (i), (ii) and (iii) of Theorem 3.1. A set of elements of $B$ that is linearly independent modulo $\left\{B_{2}, \cdots, B_{n}\right\}$ is both a set of original commutators of weight 1 and a set of nonoriginal commutators of weight 1 . We shall order this set arbitrarily. Suppose that all original and all nonoriginal commutators of weight less than $l$ have already been constructed and ordered so that whenever $s<t$

\section{"commutator of weight $s$ " $<$ "commutator of weight $t$."}

$b$ is said to be a nonoriginal commutator of weight $l$ if it is a basic commutator on the original commutators of weight less than $l$ and $b$ belongs to $\left\{B_{l}, B_{l+1}, \cdots, B_{n}\right\}$, but $b$ does not belong to $\left\{B_{l+1}, \cdots, B_{n}\right\}$. Finally it remains to define the original commutators of weight $l$. Let $B(l+1)$ denote the isolated subgroup $\left.{ }^{16}\right)$ of $B$ generated by the above nonoriginal commutators of weight $l$ and $\left\{B_{l+1}, \cdots, B_{n}\right\}$. A set of elements of $\left\{B_{l}, B_{l+1}, \cdots, B_{n}\right\}$ that is linearly independent modulo $B(l+1)$ is called a set of original commutators of weight $l$.

In passing we notice the following properties of the group $B$.

Lemma 3.3. Suppose that $B$ is a group that is generated by $n$ subgroups $B_{1}, B_{2}, \cdots, B_{n}$ which satisfy the conditions (i), (ii) and (iii) of Theorem 3.1; then we are able to make the following assertions.

(a) Let $b_{\alpha(l)}$ be a typical element of a fixed set of free generators of $B_{l}(l=1,2, \cdots, n)$. Then every element of $B$ can be represented uniquely as $a$ finite ordered product of the form

$$
\prod_{\nu} b_{\nu}^{e},
$$

where $\epsilon_{\nu}$ are integers. Further $\nu<\nu^{\prime}$ implies that if $b_{\nu}$ and $b_{\nu^{\prime}}$ are free generators of $B_{i}$ and $B_{j}$ respectively, then $i \leqq j$.

(b) $B$ is a torsion-free nilpotent group of class not exceeding $n$.

Proof. (a) It is easy to see that $\left\{B_{l}, B_{l+1}, \cdots, B_{n}\right\}$ is a normal subgroup of $B$ for all $l$. Hence considering $B$ modulo this group for $l=2,3, \cdots, n$, we obtain the above representation. The uniqueness of this representation is derived in a similar manner. It is of relevance to note that

$$
\left\{B_{l}, B_{l+1}, \cdots, B_{n}\right\}=B_{l} \cdot\left\{B_{l+1}, \cdots, B_{n}\right\}
$$

for $l=1,2, \cdots, n-1$.

(b) Using $[9$, Lemma 2.3] it follows that

$$
[B, B] \leqq\left\{B_{2}, B_{3}, \cdots, B_{n}\right\},
$$

(16) It is easily seen that $B(l+1)$ is normal in $B$ and that $\left\{B_{l+1}, \cdots, B_{n}\right\}$ is already isolated in $B$ (cf. Kuros $[6, \S 67])$. 
because of condition (ii). Similarly it follows in general that

$$
{ }^{i} B \leqq\left\{B_{i}, B_{i+1}, \cdots, B_{n}\right\}
$$

for $i<n$, and thus ${ }^{n+1} B=1$. Hence $B$ is nilpotent of class not exceeding $n$. The fact that $B$ is torsion-free follows from (a).

Let $G$ be the free $n$th nilpotent group freely generated by $a_{\alpha}, \alpha \in M$. Then $G$ can be embedded in the free $n$th nilpotent Lie algebra $\&$ over the rationals, which is freely generated by $a_{\alpha}, \alpha \in M$. In fact, the subgroup generated in $\mathscr{L}$ by $a_{\alpha}, \alpha \in M$, using Campbell-Hausdorff multiplication, is the group $G$. Further, by Lazard [7], a group $B$ that has a generating set of $n$ subgroups, which satisfy conditions (i), (ii) and (iii) of Theorem 3.1, can always be embedded in an $n$th nilpotent Lie algebra over the rationals. This follows because $B$ is a torsion-free nilpotent group of class $\left({ }^{17}\right)$ not exceeding $n$ and hence has a $\mathrm{Mal}^{\prime}$ cev completion. It only remains to apply Theorem 4.15 of Lazard [7] to the $\mathrm{Mal}^{\prime} \mathrm{cev}$ completion of $B$.

THEOREM 3.4. Let $B$ be a subgroup of a free nth nilpotent group $G$. Then $B$ can be generated by $a$ set of $n$ subgroups $B_{1}, B_{2}, \cdots, B_{n}$, where

(i) $B_{l}$ is a free nilpotent group of class $[n / l](l=1,2, \cdots, n)$;

(ii) $\left[B_{i}, B_{j}\right] \leqq\left\{B_{i+j}, \cdots, B_{n}\right\}$ if $i+j \leqq n$, and

$$
\left[B_{i}, B_{j}\right]=1 \text {, if } i+j>n \text {; }
$$

(iii) $B_{l} \cdot\left\{B_{m+1}, \cdots, B_{n}\right\} /\left\{B_{m+1}, \cdots, B_{n}\right\}$ is a free nilpotent group of class $[m / l]$, freely generated by the images of the free generators of $B_{l}$, for $m=l, l+1, \cdots, n-1$ and $l=1,2, \cdots, n-1$;

(iv) There exists a set $\left.{ }^{18}\right)$ of free generators of $B_{i}(i=1,2, \cdots, n-1)$ that has the following properties. A subset of these free generators can be taken to be the maximal set of original commutators of weight $i$ in $B$ and the images of the nonoriginal commutators of weight $l$ form a basis of the vector space $\left({ }^{19,20}\right)$

$$
\left\{\left[M\left(B_{i}\right), M\left(B_{j}\right)\right] ; i+j=l\right\} \cdot\left\{M\left(B_{l+1}\right), \cdots, M\left(B_{n}\right)\right\} /\left\{M\left(B_{l+1}\right), \cdots, M\left(B_{n}\right)\right\}
$$

for $l=2,3, \cdots, n$.

Proof. The first two conditions are the content of Theorem 3.1. The third condition is the direct consequence of Theorem 1.7. To see that the last condition holds, we consider $G$ embedded in $\mathscr{L}$ and form the Mal'cev completion of $B$. Take a set of free generators of $B_{l}$ as obtained in Theorem 3.1. Those generators which are linearly independent modulo $B(l+1)$, as defined in

(17) By Lemma 3.3 (b).

(18) Vacuous if $B_{i}$ is of rank zero and consisting of a generator of $B_{i}$ if $B_{i}$ is cyclic.

(19) Here and subsequently, if the included subgroups are $\mathrm{Mal}^{\prime} \mathrm{cev}$ completions, then \{\} will always denote the divisible subgroup (subalgebra) generated by these subgroups (subalgebras).

(20) We adopt the following conventions: $\left\{M\left(B_{n+1}\right), \cdots, M\left(B_{n}\right)\right\}=1$ and $M(\{1\})=1$. 
Definition 3.2, will form the required maximal set of original commutators of weight $l$. They can also be taken to be the maximal set of original monomials of weight $l$ of $M(B)$, because of the following three facts. If $H$ is a subgroup of $G$ and $I(H)$ denotes the isolator of $H$, then $I(H)=M(H) \cap G$. As follows from Lazard [7, Theorem 4.9.]

$$
M\left(B \cap{ }^{l} G\right)=M(B) \cap M\left({ }^{l} G\right) .
$$

Lastly, if a set of elements is a maximal set of linearly independent elements, then this is not effected by taking the Mal'cev completion of the group. We now have that by Corollary 2.3.2, the nonoriginal commutators of weight $l$ form a basis for

$$
\left\{\left(M\left(B_{i}\right), M\left(B_{j}\right)\right) ; i+j=1\right\}=\left\{\left[M\left(B_{i}\right), M\left(B_{j}\right)\right] ; i+j=1\right\}
$$

modulo ${ }^{l+1} \mathfrak{L}=M\left({ }^{l+1} G\right)$. We can, in fact, consider the concepts of original (nonoriginal) commutator and original (nonoriginal) monomial to be equivalent in our calculations. For in the formula that defines Campbell-Hausdorff multiplication, it is only the initial nonzero term which is of significance in our situation. Hence ()$^{)}$and [ ] are interchangeable. Finally as $\left\{\left[M\left(B_{i}\right), M\left(B_{j}\right)\right] ; i+j=l\right\}$ is a subgroup of $M(B)$, we can consider it modulo

$$
M(B) \cap M\left({ }^{l+1} G\right)=M\left(B \cap{ }^{l+1} G\right)=\left\{M\left(B_{l+1}\right), \cdots, M\left(B_{n}\right)\right\} .
$$

These latter equalities can easily be seen to hold if one uses a result similar to Lemma 3.3 (a), namely, Lemma 3.6, which we find more convenient to state at a later stage.

Next we examine more closely the significance of the four conditions of the above theorem.

LeMmA 3.5. Let $B$ be a group having a generating set of $n$ subgroups $B_{1}, B_{2}$, $\cdots, B_{n}$ which satisfy conditions (i), (ii) and (iii) of Theorem 3.4. Then

(a) $M\left(\left\{B_{l}, B_{l+1}, \cdots, B_{n}\right\}\right)=\left\{M\left(B_{l}\right), M\left(B_{l+1}\right), \cdots, M\left(B_{n}\right)\right\}$

$$
\left[M\left(B_{i}\right), M\left(B_{j}\right)\right] \leqq\left\{M\left(B_{i+j}\right), \cdots, M\left(B_{n}\right)\right\} \quad \begin{array}{rr}
\text { for } l=1,2, \cdots, n ; \\
\text { if } i+j \leqq n,
\end{array}
$$

and

$$
\left[M\left(B_{i}\right), M\left(B_{j}\right)\right]=1 \quad \text { if } i+j>n ;
$$

(c) The natural homomorphism

$$
B_{l} \cdot\left\{B_{m+1}, \cdots, B_{n}\right\} \rightarrow B_{l} \cdot\left\{B_{m+1}, \cdots, B_{n}\right\} /\left\{B_{m+1}, \cdots, B_{n}\right\}
$$

extends uniquely to the homomorphism $\left({ }^{21}\right)$

(21) $\left\{B_{n+1}, \cdots, B_{n}\right\}=1$. 
$M\left(B_{\imath}\right) \cdot M\left(\left\{B_{m+1}, \cdots, B_{n}\right\}\right) \rightarrow M\left(B_{l} \cdot\left\{B_{m+1}, \cdots, B_{n}\right\} /\left\{B_{m+1}, \cdots, B_{n}\right\}\right)$ with kernel $M\left(\left\{B_{m+1}, \cdots, B_{n}\right\}\right)$ for $m=l, \cdots, n$;

(d) The natural homomorphism of

$$
\left\{\left[B_{i}, B_{j}\right] ; i+j=l\right\} \cdot\left\{B_{l+1}, \cdots, B_{n}\right\}
$$

onto

$$
\left\{\left[B_{i}, B_{j}\right] ; i+j=l\right\} \cdot\left\{B_{l+1}, \cdots, B_{n}\right\} /\left\{B_{l+1}, \cdots, B_{n}\right\}
$$

extends uniquely to the homomorphism of

$$
\left\{\left[M\left(B_{i}\right), M\left(B_{j}\right)\right] ; i+j=l\right\} \cdot\left\{M\left(B_{l+1}\right), \cdots, M\left(B_{n}\right)\right\}
$$

onto

$$
\left.M\left(\left\{B_{i}, B_{j}\right] ; i+j=l\right\} \cdot\left\{B_{l+1}, \cdots, B_{n}\right\} /\left\{B_{l+1}, \cdots, B_{n}\right\}\right)
$$

with kernel $\left\{M\left(B_{l+1}\right), \cdots, M\left(B_{n}\right)\right\}$ for $l=2, \cdots, n$.

Proof. This is by induction on $n$. Results are either trivially true or vacuous for $n=1$. Consider the group

$$
B / B_{n}=\left\{B_{1} \cdot B_{n} / B_{n}, B_{2} \cdot B_{n} / B_{n}, \cdots, B_{n-1} \cdot B_{n} / B_{n}\right\} .
$$

It is easy to verify that conditions (i), (ii) and (iii) of Theorem 3.4 hold for this group. Hence, by the induction hypothesis, the above results (a), (b), (c) and (d) can be assumed to be true for $B / B_{n}$. By Lazard [7, Theorem 4.10], the natural homomorphism of $B$ onto $B / B_{n}$ gives rise to the isomorphism

$$
M(B) / M\left(B_{n}\right) \cong M\left(B / B_{n}\right) \text {. }
$$

Under this isomorphism

$$
M\left(B_{l} \cdot B_{n}\right) / M\left(B_{n}\right) \cong M\left(B_{l} \cdot B_{n} / B_{n}\right) .
$$

Now, by Lazard [7, Theorem 4.9], $M\left(B_{l} \cdot B_{n}\right)=M\left(B_{l}\right) \cdot M\left(B_{n}\right)$, as some integer power of every element of $M\left(B_{l}\right) \cdot M\left(B_{n}\right)$ belongs to $B_{l} \cdot B_{n}$ and $M\left(B_{l}\right) \cdot M\left(B_{n}\right)$ is divisible. It is more convenient to consider the group $M(B) / M\left(B_{n}\right)$ which is equal to

$$
\left\{M\left(B_{1}\right) \cdot M\left(B_{n}\right) / M\left(B_{n}\right), \cdots, M\left(B_{n-1}\right) \cdot M\left(B_{n}\right) / M\left(B_{n}\right)\right\}
$$

by the induction hypothesis.

(a) By the induction hypothesis, $M\left(\left\{B_{l} \cdot B_{n} / B_{n}, \cdots, B_{n-1} \cdot B_{n} / B_{n}\right\}\right)=\left\{M\left(B_{l} \cdot B_{n} / B_{n}\right), \cdots, M\left(B_{n-1} \cdot B_{n} / B_{n}\right)\right\}$. Hence by the above given isomorphism

$$
M\left(\left\{B_{l}, \cdots, B_{n}\right\}\right) / M\left(B_{n}\right)=\left\{M\left(B_{l}\right), \cdots, M\left(B_{n}\right)\right\} / M\left(B_{n}\right)
$$

which gives the required result on equating numerators. 
(b) As $B_{n}$ is contained in the centre of $B$, it follows that (22)

$$
M\left(B_{n}\right) \leqq M(\mathrm{Z}(B))=\mathrm{Z}(M(B)) .
$$

By the induction hypothesis, if $i+j \leqq n$, then

Also

$$
\begin{aligned}
& {\left[M\left(B_{i}\right) \cdot M\left(B_{n}\right) / M\left(B_{n}\right), M\left(B_{j}\right) \cdot M\left(B_{n}\right) / M\left(B_{n}\right)\right]} \\
& \quad \leqq\left\{M\left(B_{i+j}\right) \cdot M\left(B_{n}\right) / M\left(B_{n}\right), \cdots, M\left(B_{n-1}\right) \cdot M\left(B_{n}\right) / M\left(B_{n}\right)\right\} \\
& \quad=\left\{M\left(B_{i+j}\right), \cdots, M\left(B_{n-1}\right), M\left(B_{n}\right)\right\} / M\left(B_{n}\right) .
\end{aligned}
$$

$\left[M\left(B_{i}\right) \cdot M\left(B_{n}\right) / M\left(B_{n}\right), M\left(B_{j}\right) \cdot M\left(B_{n}\right) / M\left(B_{n}\right)\right]=\left[M\left(B_{i}\right), M\left(B_{j}\right)\right] \cdot M\left(B_{n}\right) / M\left(B_{n}\right)$, since $M\left(B_{n}\right)$ lies in the centre of $M(B)$. The required result follows now from the resulting inclusion of numerators.

It remains to consider the case $i+j>n$. It follows from the induction hypothesis that

$$
\left[M\left(B_{i}\right), M\left(B_{j}\right)\right] \leqq \mathrm{Z}(M(B)) .
$$

Let $x$ and $y$ be arbitrary elements of $M\left(B_{i}\right)$ and $M\left(B_{j}\right)$ respectively. By the definition of $\mathrm{Mal}^{\prime} \mathrm{cev}$ completions, there exist positive integers $m$ and $n$ such that $x^{m}$ and $y^{n}$ belong to $B_{i}$ and $B_{j}$ respectively. Hence

$$
[x, y]^{m n}=\left[x^{m}, y^{n}\right]=1 \text {, }
$$

since we are given that $\left[B_{i}, B_{j}\right]=1$. As $\left[M\left(B_{i}\right), M\left(B_{j}\right)\right]$ is torsion-free, we have that $[x, y]=1$ and thus $\left[M\left(B_{i}\right), M\left(B_{j}\right)\right]=1$ for $i+j>n$.

Results (c) and (d) follow easily from the induction hypothesis if one uses the isomorphism given in the initial stages of this proof. The only exception to this is the result

$$
M\left(\left\{\left[B_{i}, B_{j}\right] ; i+j=n\right\}\right)=\left\{\left[M\left(B_{i}\right), M\left(B_{j}\right)\right] ; i+j=n\right\} .
$$

This follows by Lazard [7, Theorem 4.9], as some integer power of every element of $\left\{\left[M\left(B_{i}\right), M\left(B_{j}\right)\right] ; i+j=n\right\}$ belongs to $\left\{\left[B_{i}, B_{j}\right] ; i+j=n\right\}$ and $\left\{\left[M\left(B_{i}\right), M\left(B_{j}\right)\right] ; i+j=n\right\}$ is divisible.

COROLLARY 3.5.1. The rank of the free abelian group

$$
\left\{\left[B_{i}, B_{j}\right] ; i+j=l\right\} \cdot\left\{B_{l+1}, \cdots, B_{n}\right\} /\left\{B_{l+1}, \cdots, B_{n}\right\}
$$

is equal to the dimension of the vector space $\left\{\left[M\left(B_{i}\right), M\left(B_{j}\right)\right] ; i+j=l\right\} \cdot\left\{M\left(B_{l+1}\right), \cdots, M\left(B_{n}\right)\right\} /\left\{M\left(B_{l+1}\right), \cdots, M\left(B_{n}\right)\right\}$ for $l=2,3, \cdots, n$.

In particular, we have

${ }^{(22)}$ Cf. for instance the theorem of Federov (Kuros $[6,867]$ ). 
COROLlARY 3.5.2. The rank of the free abelian group

$$
\left\{\left[B_{i}, B_{j}\right] ; i+j=n\right\}
$$

is equal to the dimension of the vector space

$$
\left\{\left[M\left(B_{i}\right), M\left(B_{j}\right)\right] ; i+j=n\right\} .
$$

Adopting a similar procedure to that given in the proof of Lemma 3.3, we can now show that the following lemma, which we used at the end of the proof of Theorem 3.4, holds.

LEMmA 3.6. Let $B$ be a group generated by the subgroups $B_{1}, B_{2}, \cdots, B_{n}$ which satisfy conditions (i), (ii) and (iii) of Theorem 3.4. If $b_{\alpha(l)}$ is a typical element of a fixed set $\left.{ }^{23}\right)$ of free generators of $B_{l}(l=1,2, \cdots, n)$, then every element of $M(B)$ can be represented uniquely as a finite ordered product of the form

$$
\prod_{p} b_{\nu}
$$

where $\epsilon_{\nu}$ are rational numbers. Further $\nu<\nu^{\prime}$ implies that if $b_{\nu}$ and $b_{\nu}$, are free generators of $B_{i}$ and $B_{j}$ respectively, then $i \leqq j$.

We are now in a position to establish the following partial converse to the above Theorem 3.4.

THEOREM 3.7. Let $B$ be a group generated by a set of $n$ subgroups $B_{1}, B_{2}, \cdots$, $B_{n}$ which satisfy the conditions of Theorem 3.4. Suppose further that the torsion subgroup of

$$
B_{l} \cdot\left\{B_{l+1}, \cdots, B_{n}\right\} /\left(\left\{\left[B_{i}, B_{j}\right] ; i+j=l\right\} \cdot\left\{B_{l+1}, \cdots, B_{n}\right\}\right)
$$

has finite exponent for $l=2,3, \cdots, n$. Then $B$ is isomorphic to a subgroup of a free nth nilpotent group.

Proof. This proceeds by induction on $n$. In fact, we shall prove more and our induction hypothesis will be as follows. Suppose that the above theorem is true for all groups $\left\{B_{1}, B_{2}, \cdots, B_{r}\right\}$, where $r<n$, and the generating subgroups satisfy the four conditions of the above mentioned type. Further we assume that the isomorphism $\psi$, which maps $\left\{B_{1}, B_{2}, \cdots, B_{r}\right\}$ into a free $r$ th nilpotent group $H$, induces the following isomorphisms into

$$
\left\{B_{m}, \cdots, B_{r}\right\} \rightarrow^{m} H \text { and }
$$

$$
\left\{B_{1}, \cdots, B_{r}\right\} /\left\{B_{m}, \cdots, B_{r}\right\} \rightarrow \psi\left(\left\{B_{1}, \cdots, B_{r}\right\}\right) \cdot{ }^{m} H /{ }^{m} H
$$

for $m=1,2, \cdots, r$. This result is well known to be true for $n=1$.

We consider now the group $B=\left\{B_{1}, B_{2}, \cdots, B_{n}\right\}$, where $B$ can be assumed to be nonabelian. It can easily be verified that the group

(23) Vacuous if $B_{l}$ is the trivial group. 


$$
B / B_{n}=\left\{B_{1} \cdot B_{n} / B_{n}, \cdots, B_{n-1} \cdot B_{n} / B_{n}\right\}
$$

satisfies the four conditions of Theorem 3.4. Here it is necessary to use the fact that the natural homomorphism

$$
B_{l} \cdot B_{n} \rightarrow B_{l} \cdot B_{n} / B_{n}
$$

extends uniquely to the homomorphism $\left({ }^{24}\right)$

$$
M\left(B_{l}\right) \cdot M\left(B_{n}\right) \rightarrow M\left(B_{l} \cdot B_{n} / B_{n}\right)
$$

with kernel $M\left(B_{n}\right)$. Hence, by the induction hypothesis, there exists an isomorphism $\phi$ that maps $B / B_{n}$ into a subgroup $\bar{A} / Z(\bar{A})$ of a free $(n-1)$ th nilpotent group $A / Z(A)$. It is convenient to take $A$ to be a free $n$th nilpotent group freely generated by $a_{\alpha}, \alpha \in M$, while $\bar{A}$ is the free $n$th nilpotent subgroup of $A$ freely generated by $a_{\alpha}^{\eta}, \alpha \in M$. The number $\eta$ is specified as follows. Firstly we choose free generators for the subgroups $B_{l}(l=1,2, \cdots, n-1)$ in the way indicated by Theorem 3.4 (iv). Let $b_{l}$ be a free generator of $B_{l}$ which is not an original commutator of weight $l$. As the torsion subgroup

$I\left(\left\{\left[B_{i}, B_{j}\right] ; i+j=l\right\} \cdot\left\{B_{l+1}, \cdots, B_{n}\right\}\right) /\left(\left\{\left[B_{i}, B_{j}\right] ; i+j=l\right\} \cdot\left\{B_{l+1}, \cdots, B_{n}\right\}\right)$

of

$$
B_{l} \cdot\left\{B_{l+1}, \cdots, B_{n}\right\} /\left(\left\{\left[B_{i}, B_{j}\right] ; i+j=l\right\} \cdot\left\{B_{l+1}, \cdots, B_{n}\right\}\right)
$$

has finite exponent, this group can be decomposed into the direct product of its torsion subgroup and a free abelian group. Now we can use the procedure given in Definition 3.2. It is to be noted that we have replaced the nonoriginal commutators by $\left\{\left[B_{i}, B_{j}\right] ; i+j=l\right\}$. The fact that we are allowed to do this follows from condition (iv), the previously stated result that if $H$ is a subgroup of $B$ then $I(H)=M(H) \cap B$ and a result of the type given in Lemma 3.5(d). By condition (iii) for $m=l$, we have that there exists an integer $\eta(l)$ such that $b_{l}^{\eta l}$ is a product of commutators of elements of $B$. We take $\eta$ to be the lowest common multiple of $\eta(n)$ with all the integers $\eta(l)$, as $b_{l}$ varies over the free generators of $B_{l}$ that are not original commutators of weight $l$ and $l$ takes the values $2,3, \cdots, n-1$. The value of $\eta(n)$ will be fixed at the last stage of our proof. We shall choose the index set $M$ large enough to enable us to perform all the necessary embeddings. Further we assume, as we are entitled to under the induction hypothesis, that $\phi$ satisfies conditions of the type given in $(\cong)$. We shall now show how to extend $\phi$ to an isomorphism $\Phi$ of $\left\{B_{1}, B_{2}, \cdots, B_{n-1}\right\}$ into $A$.

Firstly we notice that if an element of $B$ can be expressed as a product

(21) Cf. initial part of proof of Lemma 3.5. 
$\Pi\left[b^{\prime}, b^{\prime \prime}\right]$ of commutators of elements of $B$, then its image under $\Phi$ is uniquely fixed by

$$
\Phi\left(\prod\left[b^{\prime}, b^{\prime \prime}\right]\right)=\prod\left[\phi\left(b^{\prime}\right), \phi\left(b^{\prime \prime}\right)\right] .
$$

We now define the effect of $\Phi$ on the original commutators of $B$. In the proc-

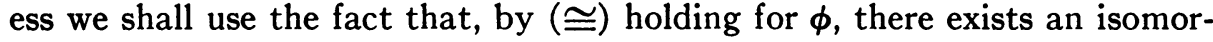
phism of

$$
\left\{B_{l}, B_{l+1}, \cdots, B_{n}\right\} /\left\{B_{l+1}, \cdots, B_{n}\right\} \text { into }{ }^{l} A /{ }^{l+1} A
$$

for $l=1,2, \cdots, n-1$. However, as at the moment our attention will be confined to the group $\bar{A}$, we are at liberty to consider the associated isomorphism of

$$
\left\{B_{l}, B_{l+1}, \cdots, B_{n}\right\} /\left\{B_{l+1}, \cdots, B_{n}\right\} \text { into }{ }^{i} \bar{A} /{ }^{l+1} \bar{A}
$$

for $l=1,2, \cdots, n-1$. By Theorem 3.4 (iii), the original commutators of $B_{l}$ will be mapped onto free generators of $\left\{B_{l}, B_{l+1}, \cdots, B_{n}\right\}$ modulo $\left\{B_{l+1}, \cdots, B_{n}\right\}$. These will now be mapped onto linearly independent elements of ${ }^{\prime} \bar{A} /{ }^{l+1} \bar{A}$. If we take coset representatives of these elements, then, by Theorem 1.3, they freely generate a free nilpotent subgroup $\bar{A}_{l}$ of $\bar{A}$ having class $[n / l]$. In this way we construct the subgroups $\bar{A}_{1}, \bar{A}_{2}, \cdots, \bar{A}_{n-1}$ of $\bar{A}$. It remains to define the effect of $\Phi$ on those free generators $b_{l}$ of $B_{l}$ that are not original commutators. As we have already noted, $b_{l}^{(n)}$ is a product of commutators of elements of $B$, for some integer $\eta(l)$. Hence the effect of $\Phi$ on $b_{l}^{\eta(n)}$ is fixed by $\phi$. Now we have to define $\Phi\left(b_{l}\right)$ so that

$$
\Phi\left(b_{l}\right)^{\eta(l)}=\Phi\left(b_{l}^{\eta(l)}\right) .
$$

By the induction hypothesis,

$$
\phi\left(b_{l}\right)^{\eta(l)}=\Phi\left(b_{l}^{\eta(l)}\right) \cdot \mathbf{Z}(\bar{A}) .
$$

Hence for an arbitrarily fixed coset representative $\phi^{\prime}\left(b_{l}\right)$ of $\phi\left(b_{l}\right)$ there exists a fixed element $z$ of $Z(\bar{A})$ such that

$$
\phi^{\prime}\left(b_{l}\right)^{\eta(l)}=\Phi\left(b_{l}^{\eta(l)}\right) \cdot z .
$$

We now put

$$
\Phi\left(b_{l}\right)=\phi^{\prime}\left(b_{l}\right) \cdot z^{-1 / \eta(l)} .
$$

The $\eta(l)$ th root of $z^{-1}$ is a uniquely determined element of $\mathcal{Z}(A)$, because of our choice of $\eta$ and the fact that $\mathbf{Z}(A)$ is torsion-free. Further

$$
\Phi\left(b_{l}\right)^{\eta(l)}=\phi^{\prime}\left(b_{l}\right)^{\eta(l)} \cdot z^{-1}=\Phi\left(b_{l}^{\eta(l)}\right) .
$$

We can now use the relations $(\cong$ ) holding for $\phi$, the induction hypothesis and 
Theorem 1.3, to show that for a fixed $l$, the free generators of $\bar{A}_{l}$ and all the $\Phi\left(b_{l}\right)$ freely generate a free nilpotent subgroup $A_{l}$ of $A$ having class $[n / l]$. In this way we have constructed the subgroups $A_{1}, A_{2}, \cdots, A_{n-1}$. Further we put

$$
A_{n}=\left\{\left[A_{i}, A_{j}\right] ; i+j=n\right\} .
$$

$A_{n}$ lies in the centre of $A$ and hence is a free abelian group. The mapping $\Phi$ of

$$
\left\{B_{1}, B_{2}, \cdots, B_{n-1}\right\} \text { onto }\left\{A_{1}, A_{2}, \cdots, A_{n-1}\right\}
$$

can also be given in the following manner. By Lemma 3.3 (a), every element $b$ of $B_{1}, B_{2}, \cdots, B_{n-1}$ can be represented uniquely in the form

$$
b=b^{\prime} \cdot \prod_{\nu(n)} b_{\nu(n)}^{\epsilon_{\nu(n)}}
$$

where $b^{\prime}$ is a finite power product of the above free generators of the subgroups $B_{1}, \cdots, B_{n-1}$, written in this order. Further $\prod_{\nu(n)} b_{\nu(n)}^{e_{\nu}(n)}$ is a finite power product of the free generators of $\left\{\left[B_{i}, B_{j}\right] ; i+j=n\right\}$. The image of $b$ in the representation $\left(^{*}\right)$ is obtained from the same representational form by substituting the corresponding free generators of the subgroups $A_{1}, A_{2}, \cdots, A_{n-1}$, which were chosen above.

(a) We now show that this is a uniquely defined mapping. Suppose that under the above rules $b$ is mapped both onto

$$
\prod_{l=1}^{n}\left(\prod_{\nu(l)} a_{\nu(l)}^{e_{\nu}(l)}\right)
$$

and onto

$$
\prod_{l=1}^{n}\left(\prod_{p(l)} a_{p(l)}^{\delta_{p}(l)}\right)
$$

where $a_{\nu(l)}$ is a typical free generator of $A_{l}$ and the above representations are of the type given in Lemma 3.3 (a). It is, of course, necessary to verify that the conditions of Lemma 3.3 hold for the group $\left\{A_{1}, A_{2}, \cdots, A_{n}\right\}$. This, however, causes no difficulties. If we now consider the images of " $b$ reduced successively modulo

$$
\left\{B_{2}, \cdots, B_{n-1}, B_{n}\right\},\left\{B_{3}, \cdots, B_{n-1}, B_{n}\right\}, \cdots, B_{n}, "
$$

under the isomorphism $\phi$, then this gives rise to the following equalities:

$$
\epsilon_{\nu(l)}=\delta_{\nu(l)}
$$

for all $\nu(l)$ and all $l(\leqq n-1)$. Further it is easy to see that the above defined mapping gives rise to a unique image for every element of $\left\{\left[B_{i}, B_{j}\right] ; i+j=n\right\}$ and hence 


$$
\epsilon_{\nu(n)}=\delta_{\nu(n)}
$$

for all $\nu(n)$. Thus we have a uniquely defined mapping $\Phi$.

(b) The mapping $\Phi$ is one-to-one, because of the unique representation (*) and Theorem 3.4 (iv), which hold for

$$
\left\{B_{1}, B_{2}, \cdots, B_{n-1}\right\} \text { and }\left\{A_{1}, A_{2}, \cdots, A_{n-1}\right\} \text {. }
$$

It is necessary to use (iv) here in order to show that $\Phi$ maps

$$
\left\{\left[B_{i}, B_{j}\right] ; i+j=n\right\} \text { one-to-one onto }\left\{\left[A_{i}, A_{j}\right] ; i+j=n\right\} \text {. }
$$

In fact, we show that this induced mapping is an isomorphism onto. Firstly the mapping is obviously a homomorphism onto. The maximal set of original commutators of weight $l$ is mapped one-to-one onto a maximal set of original commutators of weight $l$ under $\Phi$, for $l=1,2, \cdots, n-1$. Hence, by (iv), $\Phi$ induces an isomorphic mapping of

$$
\left\{\left[M\left(B_{i}\right), M\left(B_{j}\right)\right] ; i+j=n\right\} \text { onto }\left\{\left[M\left(A_{i}\right), M\left(A_{j}\right)\right] ; i+j=n\right\} .
$$

The same must hold for $\left\{\left[B_{i}, B_{j}\right] ; i+j=n\right\}$ and $\left\{\left[A_{i}, A_{j}\right] ; i+j=n\right\}$. For, by Lemma 3.5(d), $\left\{\left[M\left(B_{i}\right), M\left(B_{j}\right)\right] ; i+j=n\right\}$ and $\left\{\left[M\left(A_{i}\right), M\left(A_{j}\right)\right] ; i+j=n\right\}$ are equal to $M\left(\left\{\left[B_{i}, B_{j}\right] ; i+j=n\right\}\right)$ and $M\left(\left\{\left[A_{i}, A_{j}\right] ; i+j=n\right\}\right)$ respectively.

(c) It remains to show that $\Phi$ preserves products. Firstly, $\Phi$ obviously induces an isomorphism on each $B_{l}$ and maps it onto $A_{l}(l=1,2, \cdots, n-1)$. Let

$$
c=c^{\prime} \cdot \prod_{\nu(n)} b_{\nu(n)}^{\delta \nu(n)}
$$

be another element of $\left\{B_{1}, B_{2}, \cdots, B_{n-1}\right\}$ in standard form $\left(^{*}\right)$, then

$$
\Phi(b \cdot c)=\Phi(b) \cdot \Phi(c) \cdot z,
$$

where $z$ is some element of $Z(A)$, since the effect of $\Phi$ reduced modulo $Z(A)$ is an isomorphism, namely, $\phi$. We proceed to show that $z=1$. This can be done as follows. Put $b \cdot c$ in the standard form (*), then apply $\Phi$ and finally reverse the process of reduction to standard form. In greater detail, let the elements of $B_{l}$ appearing in the representations for $b$ and $c$ be

$$
\prod_{\nu(l)} b_{\nu(l)}^{\varepsilon_{\nu}(l)} \text { and } \prod_{\nu(l)} b_{\nu(l)}^{\delta_{\nu}(l)}
$$

respectively, where $b_{v(l)}$ is a typical free generator of $B_{l}$. Then the element of $B_{l}$ appearing in the representation for $b \cdot c$ will be

$$
\prod_{i, \nu(l)} b_{p(l)}^{e_{\nu}(l)+\delta_{\nu}(l)} \cdot c_{i}^{\alpha_{i}}
$$


where the $c_{i}$ are commutators of the elements $b_{\nu}^{\boldsymbol{y}}$ and all $c_{i}$ belong to $B_{l}$ but do not belong to $\left\{B_{l+1}, \cdots, B_{n}\right\}$. Hence the element of $A_{l}$ appearing in the representation for $\Phi(b \cdot c)$ will be

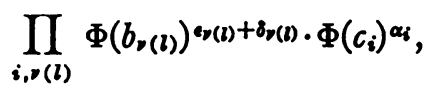

since $\Phi$ is an isomorphism on each $B_{l}$. Finally, before initiating the process of reversal, we note that the components $\Phi\left(b_{v}\right)^{\text {ev }}$ of the commutator $\Phi\left(c_{i}\right)$ can be replaced by the cosets $\Phi\left(b_{v}\right)^{e_{v}} \cdot \mathbf{Z}(A)$. Thus in the process of returning to the form

$$
\Phi(b) \cdot \Phi(c) \cdot z
$$

we are at liberty at each stage to choose the appropriate coset representative of $\Phi\left(b_{v}\right) \epsilon_{v} \cdot \mathcal{Z}(A)$, so that the same cancellations occur here as in the similar process for returning from the standard representation of the type $\left(^{*}\right)$ for $b \cdot c$ to $b \cdot c$. Hence we have that

$$
\Phi(b \cdot c)=\Phi(b) \cdot \Phi(c) .
$$

(d) We have reached the final stage of our proof. $\Phi$ has to be extended to an isomorphism $\bar{\Phi}$ of $B$ into $A$. As the torsion subgroup of

$$
B_{n} /\left\{\left[B_{i}, B_{j}\right] ; i+j=n\right\}
$$

has finite exponent $\left({ }^{25}\right)$,

$$
B_{n}=B_{n}^{*} \times B_{n}^{* *},
$$

where $B_{n}^{*}$ is the isolator of $\left\{\left[B_{i}, B_{j}\right] ; i+j=n\right\}$ and $B_{n}^{* *}$ is a free abelian group. Hence there exists a basis $b_{\tau}, \tau \in N$, of $B_{n}^{*}$ and integers $\eta_{r}, \tau \in N$, such that $b_{\tau}^{\eta_{\tau}}$ is a product of commutators for all $\tau \in N$. We fix the value of $\eta(n)$, occurring in the definition of $\bar{A}$ as a subgroup of $A$, at the lowest common multiple of the integers $\eta_{\tau}$. We can now define the effect of $\bar{\Phi}$ on $B_{n}^{*}$ :

$$
\bar{\Phi}\left(b_{\tau}\right)=\left(\Phi\left(b_{\tau}^{\eta_{\tau}}\right)\right)^{1 / \eta_{\tau}}
$$

This is a uniquely defined element of $\mathbf{Z}(A)$. For $\Phi\left(b_{\tau}^{n}\right)$ belongs to $\mathbf{Z}(\bar{A})$ and its $\eta_{\tau}$ th root exists and belongs to $\mathcal{Z}(A)$, by the choice of $\eta$; it is unique as $\mathrm{Z}(A)$ is torsion-free. This gives an isomorphism of $B_{n}^{*}$ into $\mathrm{Z}(A)$, which extends the isomorphism $\Phi$ of $\left\{\left[B_{i}, B_{j}\right] ; i+j=n\right\}$ into $Z(\bar{A})$. Now we define the effect of $\bar{\Phi}$ on $B_{l}$ to be identical with the effect of $\Phi$ on $B_{l}(l=1,2, \cdots, n-1)$, while the effect of $\bar{\Phi}$ on $B_{n}^{* *}$ can obviously be defined independently of our previous constructions. Thus we have now constructed our isomorphic mapping of $B$ into a free $n$th nilpotent group $A$. Finally it is easy to check that

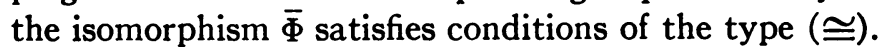

(2) Cf. Kuros $[6, \$ 29]$. 
Concluding remarks. It is of interest to note that, in Theorem 3.4 (iv), the images of the nonoriginal commutators of weight $l$ do not necessarily form a basis for the free abelian group

$$
\left\{\left[B_{i}, B_{j}\right] ; i+j=l\right\} \cdot\left\{B_{l+1}, \cdots, B_{n}\right\} /\left\{B_{l+1}, \cdots, B_{n}\right\} .
$$

Thus it is possible for

$$
\left[\left[b_{1}^{\prime}, b_{1}^{\prime \prime}\right], b_{1}^{\prime \prime \prime} 1 \equiv\left[b_{2}^{e}, b_{1}^{\prime \prime \prime}\right] \quad \text { modulo }\left\{B_{4}, \cdots, B_{n}\right\},\right.
$$

where $b_{1}^{\prime}, b_{1}^{\prime \prime}, b_{1}^{\prime \prime \prime}$ are distinct free generators of $B_{1}, b_{2}$ is a free generator of $B_{2}$ and $\epsilon$ is a positive integer. This is one of the errors which has crept into the main theorems of Goldina [1].

Condition (iii) of Theorem 3.1 for the particular case $l=1$ follows from Theorem 3.1 (ii). However, it is easy to see that, in general, condition (iii) of Theorem 3.4 does not follow from Theorem 3.4 (ii). We consider the first three particular cases of our Subgroup Theorem.

(1) $n=1$. All but one of the conditions of Theorem 3.4 are vacuous.

(2) $n=2$. Here our Subgroup Theorem reduces to a generalization of the Subgroup Theorem of Golovin and Goldina [2]. Conditions (iii) and (iv) of Theorem 3.4 are redundant.

(3) $n=3$. Here condition (iii) of Theorem 3.4 is redundant except for the particular case $l=m=2$.

It is still an open question whether conditions (i)-(iv) of Theorem 3.4 are sufficient to ensure that

$$
\left\{B_{1}, B_{2}, \cdots, B_{n}\right\}
$$

can be mapped isomorphically into a free $n$th nilpotent group, when no restrictions are placed on the torsion subgroup of

$$
\begin{gathered}
B_{e .}\left\{B_{l+1}, \cdots, B_{n}\right\} /\left(\left\{\left[B_{i}, B_{j}\right] ; i+j=l\right\} .\right. \\
\left.\quad\left\{B_{l+1}, \cdots, B_{n}\right\}\right) \text { for } l=2,3, \cdots, n .
\end{gathered}
$$

\section{REFERENCES}

1. N. P. Goldina, Free nilpotent groups, Dokl. Akad. Nauk SSSR 111 (1956), 528-530; corrigenda, 126 (1959), 694.

2. N. P. Goldina and O. N. Golovin, Subgroups of free metabelian groups, Mat. Sb. (79) 37 (1955), 323-336.

3. M. Hall, $A$ basis for free Lie rings and higher commutators in free groups, Proc. Amer. Math. Soc. 1 (1950), 575-581.

4. P. Hall, $A$ contribution to the theory of groups of prime power order, Proc. London Math. Soc. 36 (1934), 29-95.

5. - Some word-problems, J. London Math. Soc. 33 (1958), 482-496.

6. A. G. Kuros, Theory of groups, 2d ed., Chelsea, New York, 1955. 
7. M. Lazard, Sur les groupes nilpotents et les anneaux de Lie, Ann. Sci. Ecole Norm. Sup. (3) 71 (1954), 101-190.

8. A. I. Mal'cev, Two remarks on nilpotent groups, Mat. Sb. (79) 37 (1955), 567-572.

9. S. Moran, Associative operations on groups. I, Proc. London Math. Soc. (3) 6 (1956), 581-596.

10. - Unrestricted verbal products, J. London Math. Soc. 36 (1961), 1-23.

11. - Unrestricted nilpotent products, Acta Mathematica (to appear).

12. A. W. Mostowski, Nilpotent free groups, Fund. Math. 49 (1961), 259-269.

13. A. I. Siršov, Subalgebras of free Lie algebras, Mat. Sb. (75) 33 (1953), 441-452.

14. E. Witt, Treue Darstellung Liescher Ringe, J. Reine Angew. Math. 177 (1937), 152-160.

THE UnIVERSITY,

GLASGOW, SCOTLAND 